\title{
LA SECCIÓN EFICAZ EN LA ESTRUCTURA DE FÁBRICA
}

\section{(THE EFFECTIVE SECTION IN MASONRY STRUCTURE)}

\author{
Manuel J. Freire Tellado, Arquitecto \\ Dpto. de Tecnología de la Construcción. Univ. de La Coruña.
}

ESPAÑA

Fecha de recepción: 25-IV-97

631-16

RESUMEN

Los graves problemas de las estructuras de fábrica para resistir los esfuerzos de tracción han dado lugar a distintos métodos de cálculo para validar las secciones resistentes en estudio, tratando de buscar concordancia entre sus resultados y los arrojados por la experiencia práctica.

En este estudio se analiza el método de la Sección Eficaz, estableciendo tanto los distintos tipos de ésta que pueden darse para una sección rectangular como las condiciones que discriminan la respuesta de la sección de acuerdo con uno de los tipos anteriores. Se analiza también la influencia de este método de cálculo sobre el Eurocódigo 6.

Por último, se proponen un conjunto de diagramas adimensionales que permiten la verificación de la sección de acuerdo con las distintas excentricidades que pueden darse en la aplicación del axil. Dentro de estos diagramas se reflejan los distintos métodos de validación de la sección en estudio, de tal forma que se permite una sencilla comparación entre los resultados arrojados por distintos métodos de cálculo. Posteriormente estos diagramas se vuelcan como ábacos adimensionales de comprobación de la sección.

\section{SUMMARY}

The incapability of masonry structures to bear tension have led to the development of a number of specific calculus methods in order to reproduce the results of practice experience.

This paper analyzes the Effective Section Method proposed by the spanish standard FL-90 and its influence on Eurocode 6. Various kinds of resistant sections for a rectangular element in shape are established. Boundaries between these types are also determined.

At last a number of diagrams are proposed in order to test the section under different loads. These diagrams are the result of the employ of various calculus methods. Therefore they are conceived as calculus non dimensional abacous.
Desde los tiempos más remotos se plantea la pregunta de cómo determinar el espesor mínimo de un muro para que éste resulte estable. Hasta la aparición de los distintos métodos de cálculo, allá por el siglo XIX, esta pregunta se resolvió con el método de prueba y error, que se fue concretando en un conjunto de normas prácticas.

Hoy en día, el mundo de la estructura de fábrica de ladrillo se rige en España por la norma NBE-FL-90, Muros Resistentes de Fábrica de Ladrillo [1], actualización y ligera corrección de la anterior $M V-201-1972$ [2], que, a su vez, tiene su origen en el pliego PIET-70 del I.E.T.C.C. [3]. En fechas recientes -marzo 1997- se ha publicado en España la norma experimental UNE-ENV 1996-1-1, traducción al castellano del Eurocódigo 6. Proyecto de Estructuras de Fábrica, norma, como se ha dicho, experimental y que constituirá la referencia europea en un futuro no lejano [4].

La norma NBE-FL-90 admite como válidos distintos métodos de comprobación de la sección, proponiendo ella misma el método conocido como Método de la Sección 
Eficaz. Admite implícitamente, asimismo, el método elástico lineal, con una limitación de la resistencia a tracción de la fábrica limitada a la décima parte de su resistencia a compresión. Sin embargo, éstos son sólo algunos de los métodos planteados para el estudio resistente de las secciones de fábrica. D. José María Jenaro Garrido [5] recoge varios diagramas de cálculo para la fábrica, entre ellos el de distribución parabólica de las tensiones de compresión sobre la pieza.

Toda situación que implique la aparición de un esfuerzo axil deviene, en la práctica, en una situación de compresión compuesta, flexocompresión, flexotracción o tracción compuesta -debido al tipo de axil actuante, tracción o compresión, y a la proporción de su magnitud con la del esfuerzoflector actuante- a causa de las excentricidades que se originan en la actuación de este esfuerzo con respecto al c.d.g. de la sección, excentricidades que pueden ser debidas a una aplicación excéntrica del axil, a la actuación de esfuerzos horizontales, a eventuales imperfecciones en la ejecución, así como también a variaciones locales del módulo de elasticidad del material (anisotropía) que signifiquen variaciones de la posición del c.d.g. de la sección a lo largo de la directriz de la pieza.

Estas excentricidades en la aplicación de la carga conducen a la conocidísima condición de agotamiento elástico de una sección

$$
\sigma=\frac{\mathrm{N}}{\mathrm{A}}+\frac{\mathrm{M}_{1}}{\mathrm{~W}_{1}}+\frac{\mathrm{M}_{2}}{\mathrm{~W}_{2}} \leq \sigma_{\mathrm{d}}
$$

donde obviamente, para un material elástico, la tensión sobre la sección (tracción o compresión) que resulte determinante será del mismo signo que el esfuerzo axil aplicado.

Si reducimos el estudio a la aplicación de esfuerzos axiles de compresión -a los que se les asignará el signo ' + ' de acuerdo con el criterio de la norma NBE-FL-90, Muros Resistentes de Fábrica de Ladrillo-, las dos condiciones que engloba la fórmula anterior se pueden reescribir como

$$
\begin{gathered}
\sigma_{c}=\frac{N}{A}+\frac{M_{1}}{W_{1}}+\frac{M_{2}}{W_{2}} \leq \sigma_{d} \text { (Limitación por compresión) } \\
-\sigma_{t}=\frac{N}{A}-\frac{M_{1}}{W_{1}}-\frac{M_{2}}{W_{2}} \geq-\sigma_{d} \text { (Limitación por tracción) }
\end{gathered}
$$

entre las que resultará decisiva la primera para la comprobación de la sección. Empero, si el material no presenta un comportamiento isótropo, ambas condiciones deben ser verificadas al unísono. Cuando el material presenta una manifiesta incapacidad de resistencia a tracción, las condiciones para su dimensionado se enunciarán diciendo que el esfuerzo de compresión no supere el valor de la resistencia del material a compresión $-1^{\mathrm{a}}$ condición- y que no aparezcan tensiones de tracción sobre la sección -lo que equivale a exigir que la segunda condición no supere el valor de 0 -. Tradicionalmente esta condición se expresaba imponiendo la condición de que el axil estuviese contenido dentro del núcleo central de la sección.

Dentro del apartado de Flexión, la norma NBE-FL-90, Muros Resistentes de Fábrica de Ladrillo admite para las fábricas -con ciertas condiciones: se exige la justificación explícita y la toma de precauciones que permitan garantizar esta resistencia a tracción- un valor de tracción igual a $0,10 \cdot \sigma_{d}$, lo que lleva a las condiciones de resistencia elástica de la pieza siguientes:

$$
\begin{gathered}
\sigma_{c}=\frac{N}{A}+\frac{M_{1}}{W_{1}}+\frac{M_{2}}{W_{2}} \leq \sigma_{d} \text { (Limitación por compresión) } \\
-\sigma_{t}=\frac{N}{A}-\frac{M_{1}}{W_{1}}-\frac{M_{2}}{W_{2}} \geq-0,1 \sigma_{d} \text { (Limitación por tracción) }
\end{gathered}
$$

condiciones que establecen una doble limitación simultánea para los valores admisibles de los esfuerzos en la sección.

Dado que los momentos flectores actuantes se pueden expresar en función de las excentricidades como $M_{1}=N \cdot e_{1}$ y que $M_{2}=N \cdot e_{2}$ y como $e_{1}$ y $e_{2}$ se pueden expresar como $e_{1}=\lambda_{1} \cdot h$ y $e_{2}=\lambda_{2} \cdot b$, para una sección rectangular de dimensiones $b \times h$, lascondiciones anteriores se transforman en:

$$
\begin{gathered}
\sigma_{c}=\frac{N}{A}\left(1+6 \cdot \lambda_{1}+6 \cdot \lambda_{2}\right) \leq \sigma_{d} \\
-\sigma_{t}=\frac{N}{A}\left(1-6 \cdot \lambda_{1}-6 \cdot \lambda_{2}\right) \leq 0,1 \sigma_{d}
\end{gathered}
$$

Si, en un primer análisis, se considera que la excentricidad $e_{2}=0$, las condiciones anteriores de resistencia se pueden representar gráficamente en función de $e_{1} \mathrm{y} N$. Éste es el contenido del gráfico adimensional de la Figura 1, que presenta una asíntota horizontal.

Una estructura comola fábrica rara vez presenta continuidad con los elementos horizontales, de tal forma que los momentos introducidos en la estructura son debidos, fundamentalmente, a excentricidades en la aplicación de las cargas sobre la sección. Parece así difícil mantener que, como consecuencia de la anelasticidad del material, una sección suficiente para resistir un esfuerzo axil dado que 


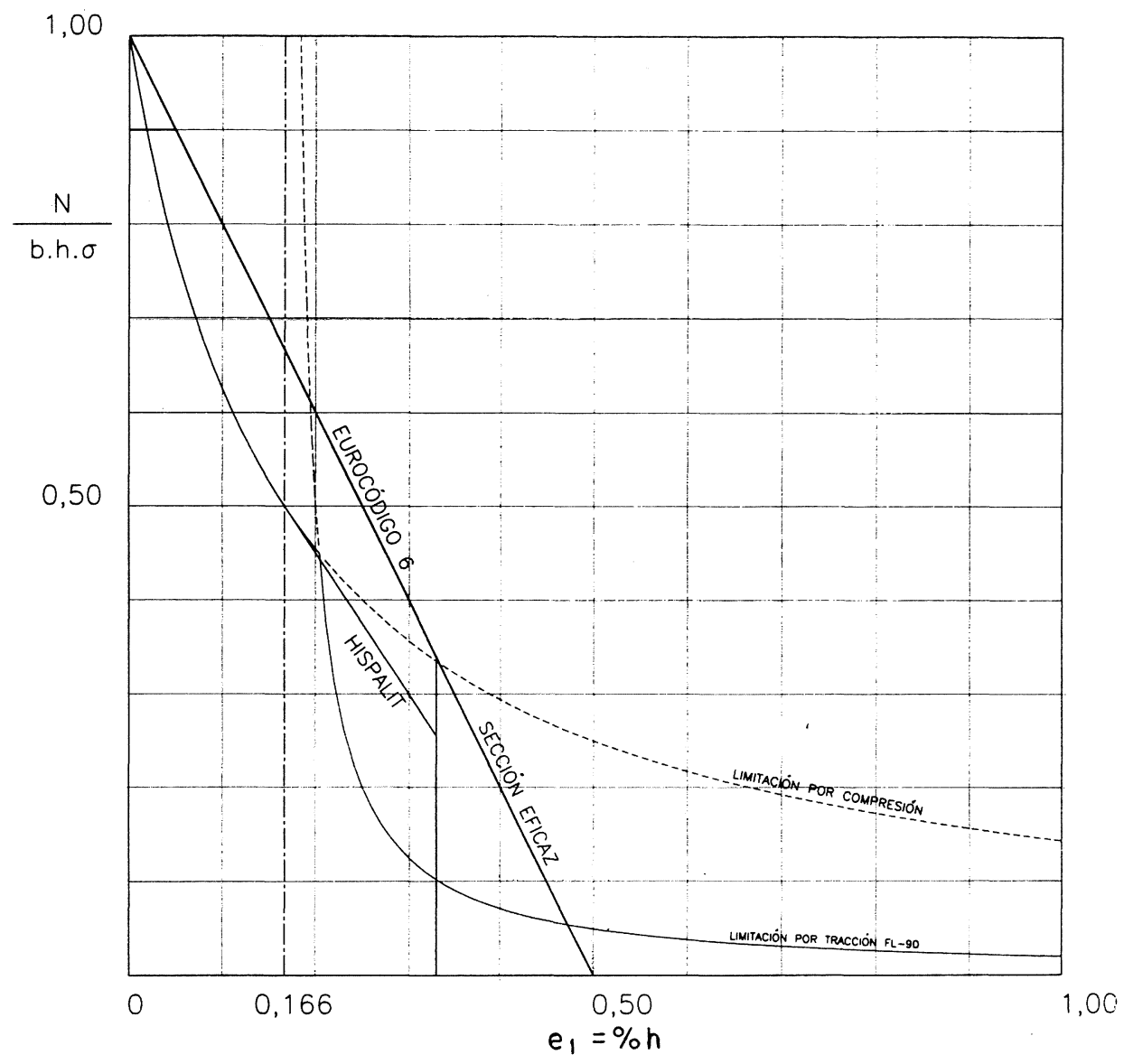

Fig. 1.- Sección rectangular sometida a compresión compuesta $\left(e_{1}=\%\right.$ h).

actuase centrado dejase de serlo-debido a la aparición sobre ella de tracciones de magnitud excesiva- al aumentar sus dimensiones -manteniendo fijo uno se sus vértices y el punto de aplicación de la carga- debido al desplazamiento del c.d.g. y a las excentricidades consecuentes. A esta objeción la norma FL-90 sale al paso, proponiendo el método de la Sección Eficaz (art. 5.4.1) [6]

Se entiende por Sección Eficaz una parte de la sección del elemento, con un área $B$, delimitada por una recta secante y cuyo baricentro coincide con el punto de aplicación del esfuerzo normal. La Sección Eficaz se considera en cálculo respondiendo a tensión uniforme, considerando inactivo el resto de la sección del elemento. La condición de seguridad expresaasí lacomprobación a compresión simple:

$$
\sigma=\frac{N_{d}}{B} \leq f_{d}
$$

Obviamente, dado que la condición expresa que la tensión que se produce sobre la Sección Eficaz sea menor que la tensión de cálculo, equivale a admitir la plastificación completa de la sección resistente. Por supuesto, si la carga se aplica centrada -esto es, su punto de aplicación coincide con el baricentro de la sección- la sección eficaz coincide con la total, $B=A$.

Las condiciones enunciadas anteriormente no son privativas de este método. En la referencia [7], el método de cálculo propuesto hace suya la condición de despreciar

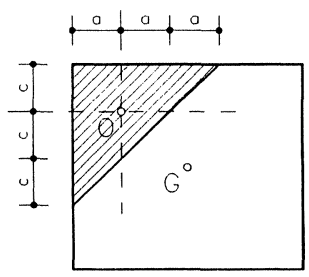

Sección eficaz de un elemento (G: BARICENTRO DE LA SECCIÓN: O: PUNTO DE APLICACIÓN DEL AXIL)

parte de la sección, pero la supone resistiendo con una respuesta elástica y lineal, de tal forma que para excentricidades inferiores a $t / 6$ la respuesta es el clásico diagrama trapezoidal, mientras que para excentricidades superiores al valor indicado la respuesta se esquematiza mediante un diagrama triangular, cuyo baricentro coincide con el punto de aplicación del axil. Se impone, además, un límite de $0,33 t$ a la excentricidad máxima, 
siendo $t$ el espesor del muro.Estas condiciones se representan en la curva rotulada como HISPALYT, dentro de la Figura 1.

Los supuestos de sección anteriores permiten una notable ampliación de los casos definidos como admisibles por el cálculo, situación que se grafia en la Figura 2 para una situación de excentricidad única -en una sola dirección del plano-. Las curvas continuas exteriores del gráfico abarcan todas las situaciones admisibles para FL-90 en la situación señalada, combinando los dos criterios de aceptación que fija la norma y que corresponden, en su mayoría, a situaciones admisibles con el criterio de la Sección Eficaz, a los que se añade el pequeño grupo de casos de excentricidad extrema que permite incluir la condición de respuesta elástica.

La Figura 1 muestra, para una solicitación de compresión compuesta en la que se ha considerado nulala excentricidad en la dirección del ancho de la pieza, las distintas limitaciones portantes de acuerdo con el método de cálculo escogido. En abscisas se representa la excentricidad -en el sentido del canto- con la que está aplicada el axil en la sección, excentricidad que se introduce como porcentaje del canto. En ordenadas se refleja el cociente entre el axil actuante, $N$, y el axil de agotamiento de la sección $(b \cdot h \cdot \sigma)$ para carga centrada. En ambos casos, las magnitudes requeridas son adimensionales. El gráfico puede usarse para el dimensionado de elementos (Figura 3), sin más que introducir los oportunos coeficientes de seguridad, tomando como $N=N_{d}$ y sustituyendo $\sigma$ por $f_{d}$.

La curva rotulada como Sección Eficaz corresponde a la pareja de valores Axil-Excentricidad que agotarian la sección con el criterio de resistencia a compresión admitido por NBE-FL-90. La curva definida como Limitación por Compresión representa los valores que producirían el agotamiento de la sección de acuerdo con la fórmula de obtención de las tensiones de compresión clásica de la Resistencia de Materiales:

$$
\sigma=\frac{M_{d}}{W}+\frac{N_{d}}{A} \leq f_{d}
$$

mientras que la curva de nombre Limitación por Tracción corresponde a la pareja que produciría el agotamiento de la sección por la aparición de tensiones de tracción, que superasen el valor tolerado por el criterio de NBE-FL-90 expresado anteriormente:

$$
\sigma=\frac{M_{d}}{W}+\frac{N_{d}}{A} \leq 0,10 f_{d}
$$

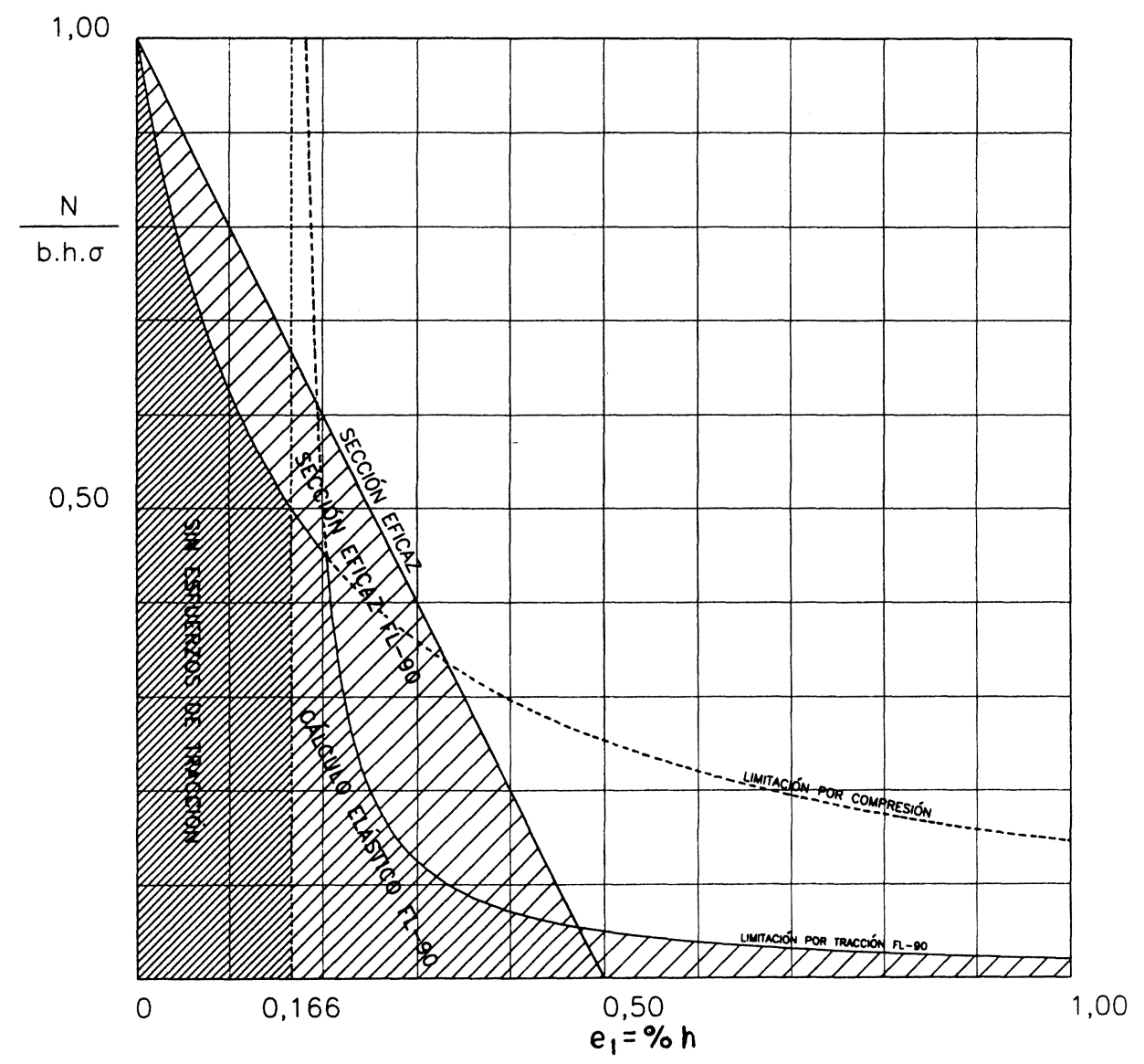

Fig. 2.- Sección rectangular sometida a compresión compuesta $(F L-90)\left(e_{l}=\%\right.$ h). 
Como se ve, esta curva admite como válidas una serie de combinaciones de alta excentricidad y escaso axil que no resultarían admisibles con el criterio de la Sección Eficaz, criterio que obviamente no permite que la excentricidad llegue a alcanzar el valor de la mitad del canto.

La línea discontinua marca la excentricidad límite, que haría que la carga estuviese aplicada fuera del tercio central $\mathrm{y}$, por tanto, el punto de aparición de tensiones de tracción en la pieza de acuerdo con las teorías clásicas de Resistencia de Materiales. Si se desea aplicar este principio, ausencia de tensiones de tracción sobre la sección, como criterio de comprobación, la zona que representaría las parejas de valores admisibles para la sección sería la delimitada por esta línea -y situada a su izquierda- y la curva de Limitación por Compresión.

De la misma forma, la aplicación del criterio de límite de compresiones, dado por la fórmula de la compresión compuesta y limitación por tracción de acuerdo con la formulación de FL-90, arrojaría como valores admisibles todos aquéllos que se encontrasen situados en la esqui- na inferior izquierda, por debajo de la gráfica de Limitación por Compresión hasta la intersección con la de Limitación por Tracción, punto, a partir del cual, ésta sería la frontera.

La comparación con estos criterios de dimensionado permite entender la notable ampliación de valores admisibles que implica el criterio de la Sección Eficaz, siempre más permisivo que cualquier otro, salvo para aquellos valores con altas excentricidades, tal y como se deriva del incremento de superficie en el gráfico que significa este criterio. Se han representado, asimismo, los casos HISPALYT ya citados anteriormente y, también, el correspondiente al Eurocódigo 6.

En el análisis anterior se ha considerado la existencia de dos excentricidades $e_{1}$ y $e_{2}$ en el sentido del canto -o espesor- $h$ del muro, la primera, $e_{1}, y$ la segunda, $e_{2}$, en el sentido del ancho $b$ del muro, tomándose $e_{2}=0$ en este primer caso. Así, para una sección rectangular de dimensiones $b \mathrm{x} h$, la condición de resistencia, según el criterio de la Sección Eficaz en la aplicación de un axil $N_{d}$

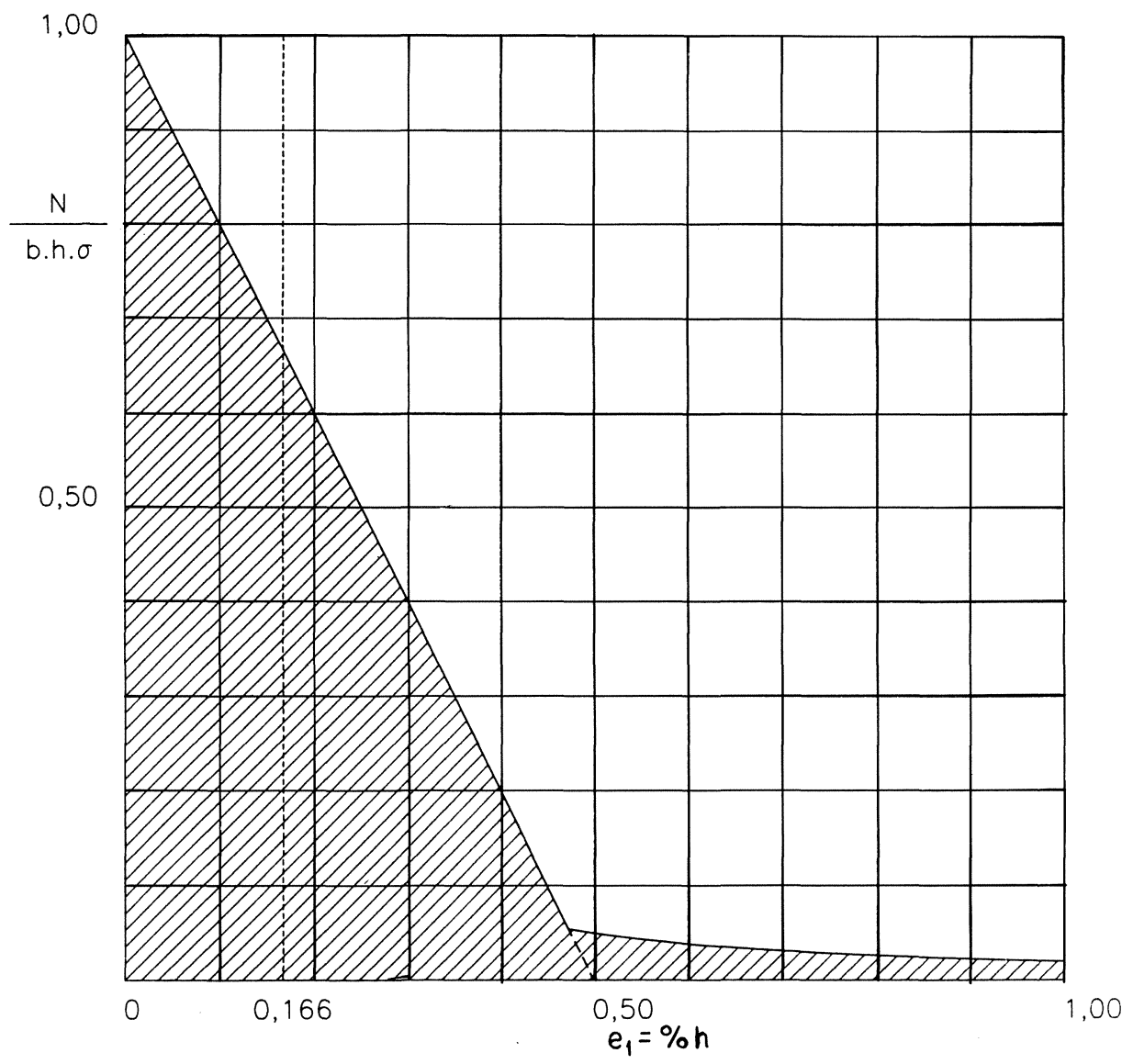

Fig. 3.- Sección rectangular sometida a compresión compuesta (diagrama de dimensionado) $\left(e_{1}=\% h\right)$. 
con una excentricidad $e_{1}$, se puede obtener como:

$$
\begin{aligned}
& \text { Área resistente } \quad \mathrm{B}=2\left(\frac{\mathrm{h}}{2}-\mathrm{e}_{1}\right)=\mathrm{h}-2 \mathrm{e}_{1} \\
& \text { Axil resistente } \mathrm{N}_{\mathrm{rd}}=\mathrm{f}_{\mathrm{d}} \cdot \mathrm{b}\left(\mathrm{h}-2 \mathrm{e}_{1}\right)=\mathrm{f}_{\mathrm{d}} \cdot \mathrm{b} \cdot \mathrm{h}\left(1-\frac{2 \mathrm{e}_{1}}{\mathrm{~h}}\right)
\end{aligned}
$$

donde $\mathrm{f}_{\mathrm{d}}=\mathrm{f}_{\mathrm{k}} / \gamma_{\mathrm{m}}$ es la resistencia de cálculo de la fábrica afectada del coeficiente de minoración correspondiente. Si llamamos:

$$
\theta_{\mathrm{i}}=\left(1-\frac{2 \cdot \mathrm{e}-1}{\mathrm{~h}}\right)
$$

la condición resistente puede expresarse como:

$$
\mathrm{N}_{\mathrm{sd}} \leq \mathrm{N}_{\mathrm{rd}}=\theta_{\mathrm{i}} \frac{\mathrm{b} \cdot \mathrm{h} \cdot \mathrm{f}_{\mathrm{k}}}{\gamma_{\mathrm{m}}}
$$

que es la fórmula propuesta por el Eurocódigo 6 para la comprobación de muros de fábrica, antes de aplicar la oportuna reducción de la carga soportada en función de lá esbeltez de la pieza. Esta norma añade además, como condición de mínimo, la limitación

$$
e_{1} \geq 0,05 \cdot h
$$

que da lugar al tramo horizontal que se observa en la curva del método propuesto por esta norma. Es preciso decir, asimismo, que si bien entre el articulado no aparece limitación alguna para la excentricidad máxima -que el propio método de cálculo limita a $0,50 \cdot h$-, entre las tablas que se ofrecen para facilitar los cálculos aparece como límite la relación (obsérvese la concordancia con lo planteado en la referencia [7]):

$$
\frac{\mathrm{e}}{\mathrm{h}}=0,33
$$

Pese a este método de cálculo, la norma recoge un diagrama de respuesta de la fábrica e incorpora expresamente el diagrama parabólico entre sus aproximaciones. Sin embargo, en la formulación de cálculo, olvida estos diagramas de respuesta.

La Figura 1 puede emplearse para el dimensionado de las piezas sin más que sustituir los valores de $N$ por $N_{d}$ y de $\sigma$ por $\sigma_{d}$, con la ventaja de saber las condiciones de trabajo de la sección -si se producen tracciones sobre la sección, si es admisible con comportamiento elástico...- y el método de cálculo que avala su aceptación o rechazo, criterios que posibilitan la toma de decisiones más fundadas del proyectista.

El Eurocódigo 6 no recoge método de cálculo alguno para la situación de pilastras o elementos de fábrica sometidos a la aplicación del axil con dos excentricidades perpendiculares con respecto al c.d.g. de la sección. Tampoco otras referencias, como la [7], citada anteriormente, proponen método de cálculo alguno. Por ello, en estas situaciones, nos ceñiremos a lo expuesto por la norma española NBE-FL-90, como una posible generalización del método del Eurocódigo.

La situación de la sección es de compresión compuestaesviada-actuación de dos excentricidades- loque complica notablemente el problema. De hecho, llegados a este punto la norma FL-90 establece que, si la Sección Eficaz es de difícil determinación geométrica, puede considerarse unaSección Eficaz A proximada, cuyo baricentro coincida con el punto de aplicación de la carga. Según la norma, si ésta se elige convenientemente, el error cometido es pequeño y va del lado de la seguridad, puesto que la Sección Eficaz tiene área máxima. Este caso se plantea gráficamente en la norma para una sección rectangular.

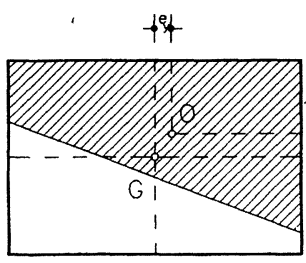

G: BARICENTRO DE LA SECCIÓN

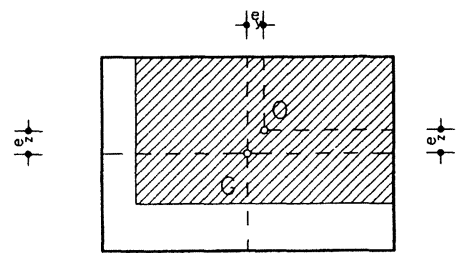

O: PUNTO APLICACIONN DEL AXIL
Sección eficaz y sección eficaz aproximada

Introducir el planteamiento de Sección Eficaz Aproxima$d a$ en el análisis de la sección sometida a un axil aplicado con dos excentricidades parece conveniente para el cálculo manual, pero deja dudas sobre las capacidades últimas que se derivan del método de laSección Eficaz. Estas dudas pretenden ser resueltas por las líneas que siguen para el caso de una sección rectangular.

De acuerdo con la definición de la norma, para las distintas combinaciones de excentricidades en la aplicación del axil se dan cuatro posibles formas de secciones eficaces sobre la pieza: Sección Eficaz Triangular, Sección Eficaz Trapezoidal de base b. Sección Eficaz Trapezoidal de base hy Sección Eficaz Pentágono-Irregular, que, junto con las Secciones Eficaces Rectangulares de las situaciones límite, constituyen las seis formas posibles de respuesta de la sección de acuerdo con la norma, que se esquematizan seguidamente.
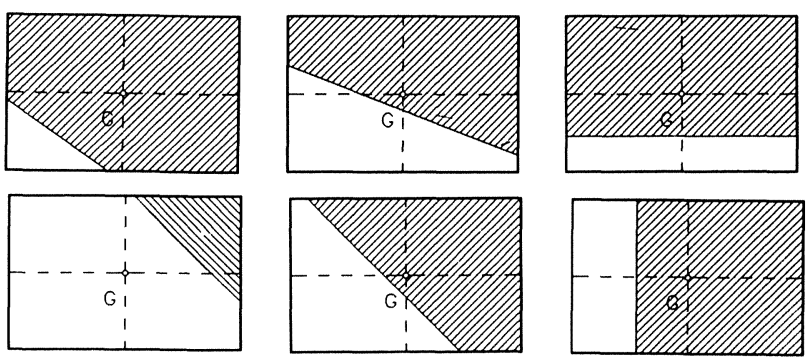

Secciones eficaces posibles en un elemento rectangular G: BARICENTRO DE LA SECCIÓN 
La Figura 4 permite diferenciar claramente las zonas de la sección que conducen a las distintas situaciones de respuesta de ésta. En general, el paso de una a otra responde a condiciones sencillas vinculadas con las coordenadas del núcleo central de la sección, salvo en el paso de la sección eficaz pentagonal a sus contiguas trapeciales, que se encuentra gobernada por una pareja de ecuaciones de tercer grado no resolubles directamente. Para la rama inferior de la curva estas ecuaciones son:

$$
\begin{gathered}
e_{1}=\frac{h^{2}}{12}\left[\frac{b_{0}}{b} \frac{\left(3 b+6 e_{2}-2 b_{0}\right)\left(6-2 e_{2}\right)}{b_{0}(3 b-2)+6 e_{2}\left(b_{0}-1\right)}-1\right] \\
b_{0}^{3}+a_{1} \cdot b_{0}{ }^{2}+a_{2} \cdot b_{0}+a_{3}=0
\end{gathered}
$$

siendo

$$
\begin{gathered}
a_{1}=1,5 \cdot h \frac{e_{2}}{e_{1}}-3 \cdot e_{2}-3 \cdot b \\
a_{2}=2,25\left(b^{2}+2 \cdot b \cdot e_{2}-b \cdot h \frac{e_{2}}{e_{1}}-2 \cdot h \frac{e_{2}}{e_{1}}\right) \\
a_{3}=6 \cdot b \cdot h \frac{e_{2}}{e_{1}}
\end{gathered}
$$

El trazado de las curvas que muestra la Figura 4 se ha resuelto por un método iterativo.
De las consideraciones anteriores se sigue que, para cada punto de aplicación del esfuerzo axil sobre la sección, es posible determinar el tipo de Sección Eficaz de respuesta de la pieza, las dimensiones de ésta y, por tanto, el valor del esfuerzo axil máximo que es capaz de soportar la pieza. De todas formas, no cabe olvidar que la Sección Eficaz será, en todo caso, la que de entre todas las configuraciones posibles proporcione unárea máxima, condición que resulta útil en programación.

Con estas premisas se ha abordado la realización de los gráficos que se acompañan. Se ha optado por dos series de gráficos, una, con excentriciades $e_{1}, e_{2}$ independientes entre sí y una segunda, en la que se ha establecido una relación porcentual entre ellas $e_{2}=k . e_{1}$. Estos dos criterios corresponden al estudio de los valores admisibles sobre la sección para una variación de las excentricidades en forma de rectas verticales -primer caso de la Figura 5- o de rectas que pasan por el c.d.g. de la sección -segundo caso-.

En la primera colección -Figuras 6 a 10- se han obtenido los valores del axil y de la excentricidad $e_{1}$ para una serie discreta de valores de la excentricidad $e_{2}(0,5,10,15$, $20,25,30,35,40 \ldots \%$ B), que se ha traducido en la curva correspondiente. Para aquellos valores de $e_{2}$ inferiores a $b / 6=16,667 \%$ se ha trazado, asimismo, la recta que marca la posición de la excentricidad $e_{1}$ que lleva el axil fuera del núcleo central de la sección, con la consiguiente aparición de tracciones según el diagrama elástico. La

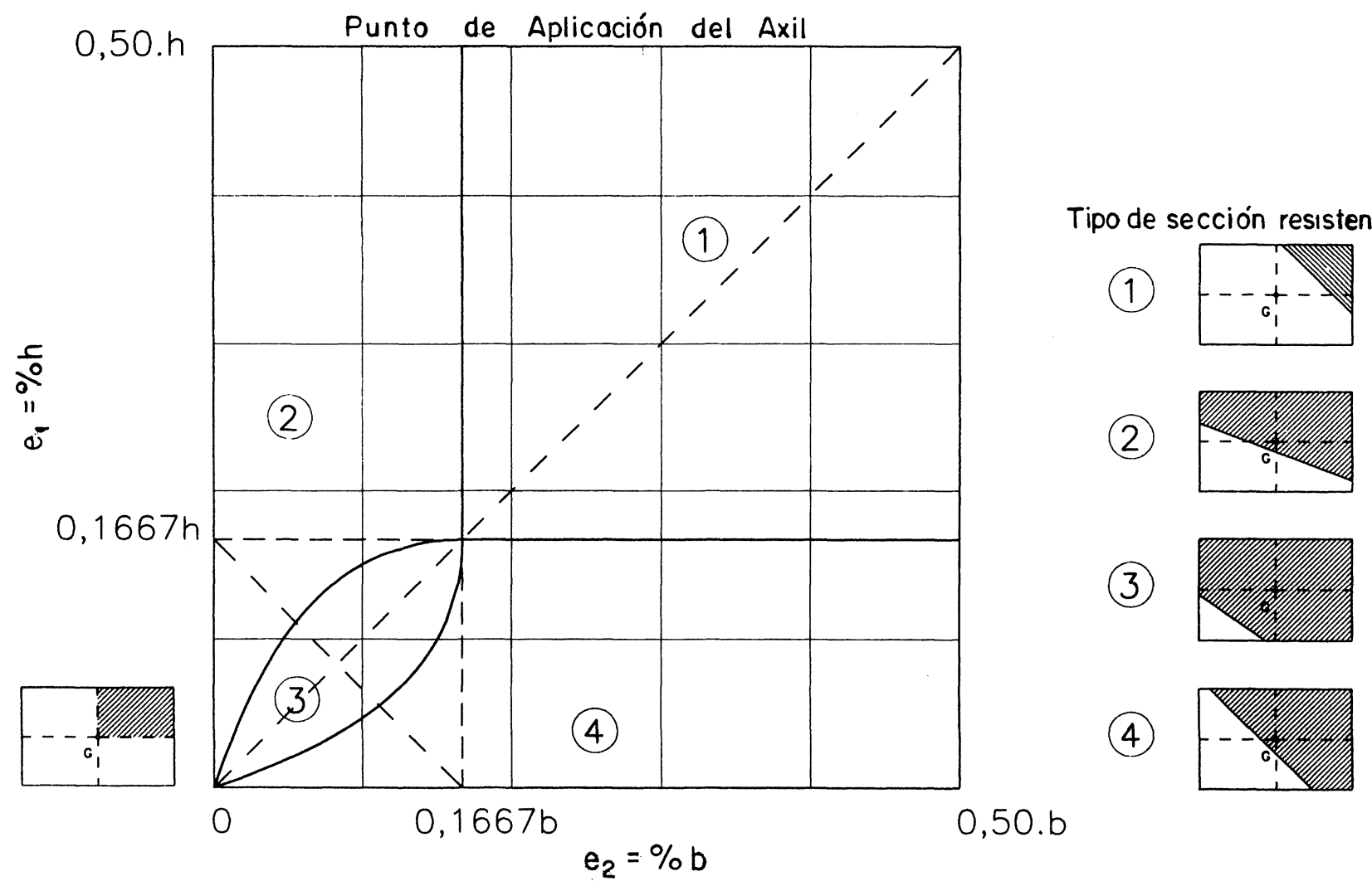

Fig. 4.- Sección rectangular sometida a compresión compuesta esviada. Punto de aplicación del axil. 
curva correspondiente a la Sección Eficaz Total va recorriendo las distintas situaciones de ésta. Como comparación se dibuja también la curva correspondiente a los valores de la Sección Eficaz Aproximada que señala la norma y las gráficas correspondientes a las condiciones de respuesta elástica indicadas anteriormente. Es de hacer notar que los valores arrojados por la Sección Eficaz Aproximada definida por la norma están más cerca de la

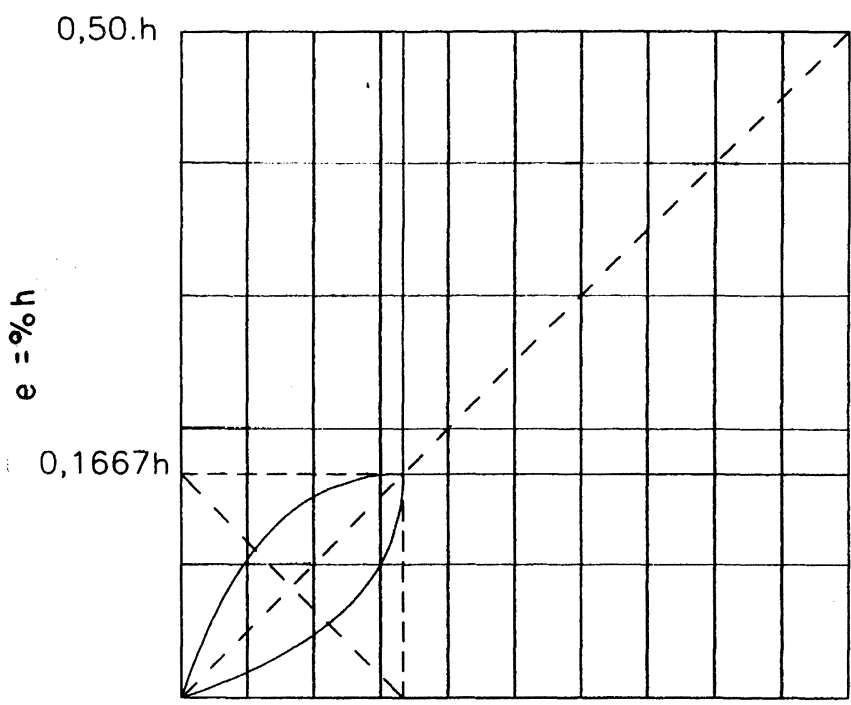

0

$0,1667 b$
$0,50 . b$

$e_{2}=\% b$
$0,50 . h$

$0,1667 h$

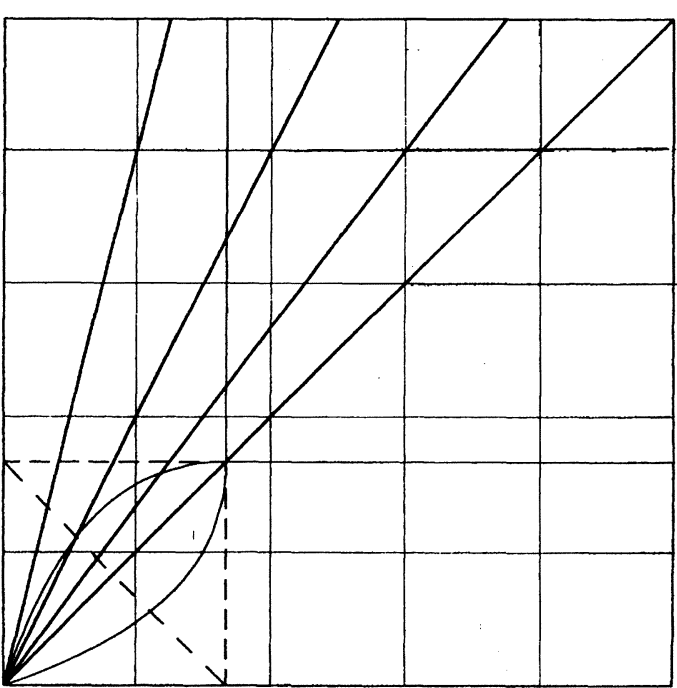

$0,1667 b$

0,50 .

Fig. 5.- Sección rectangular sometida a compresión compuesta esviada. Excentricidades aplicadas en la obtención de los diagramas.

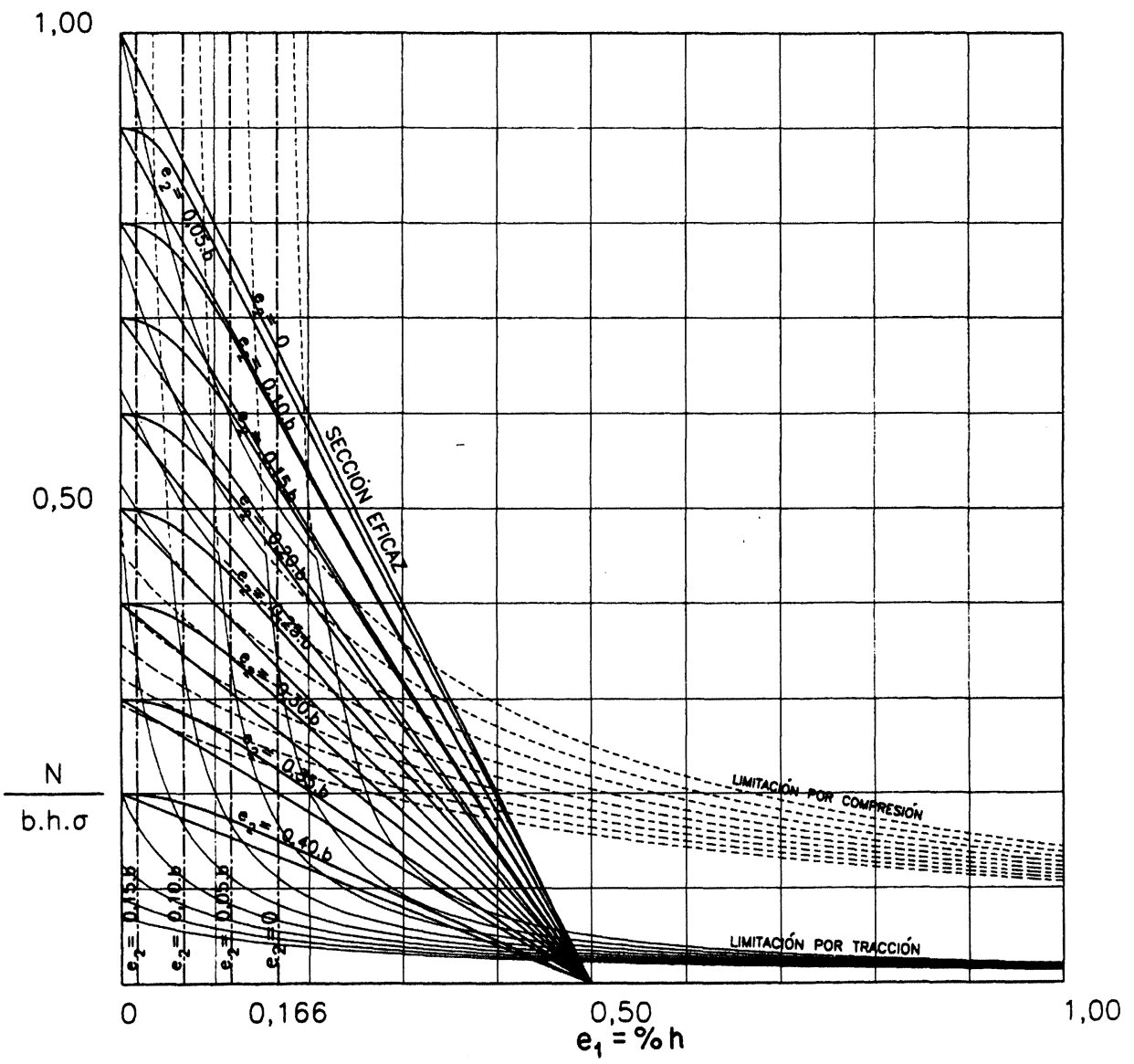

Fig. 6.- Sección rectangular sometida a compresión compuesta esviada. Valores del axil y $e_{1}$ para una serie de valores de $e_{2}$. 


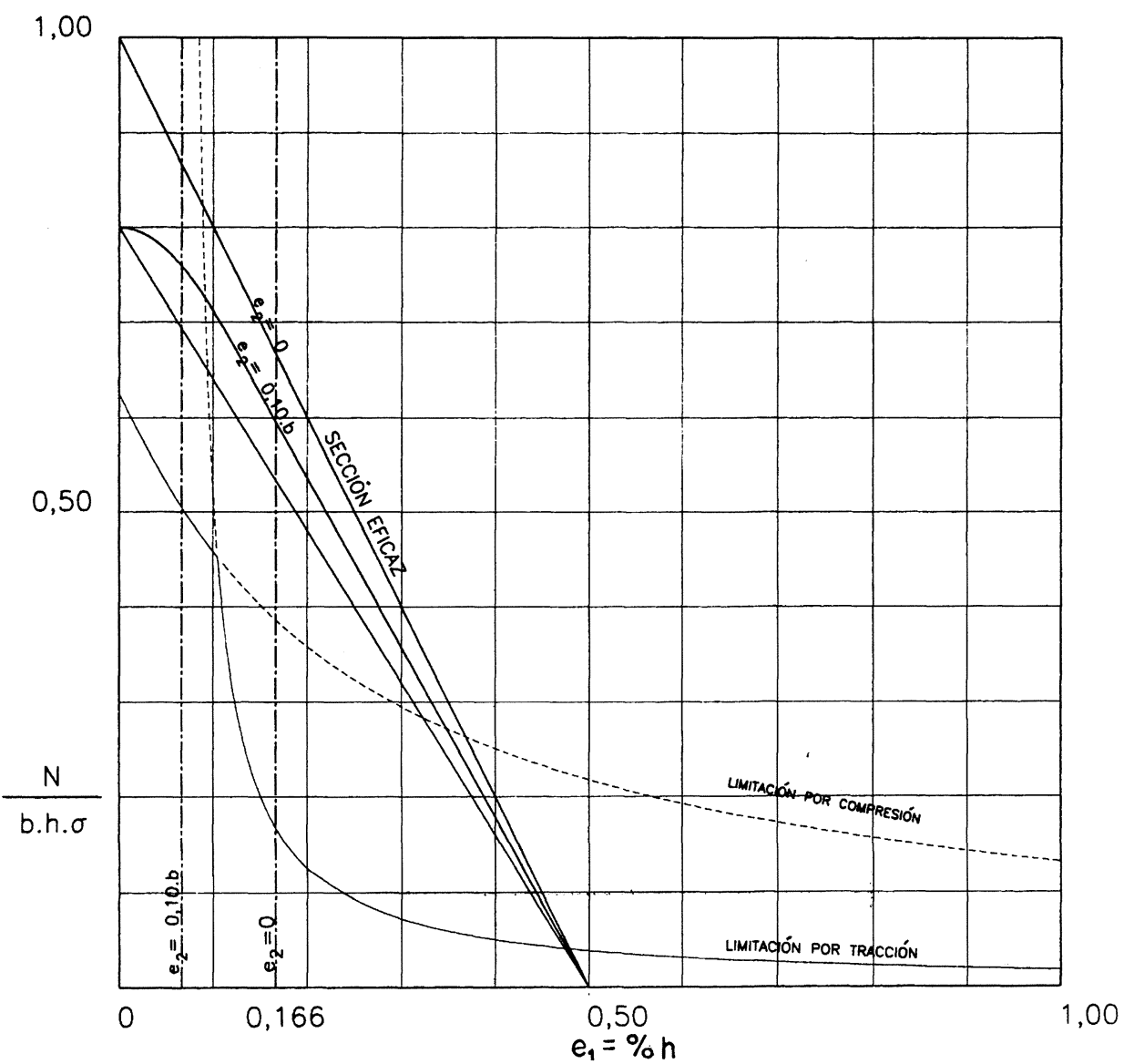

Fig. 7.- Sección rectangular sometida a compresión compuesta esviada. Valores del axil y $e_{1}$ para una serie de valores de $e_{2}$.

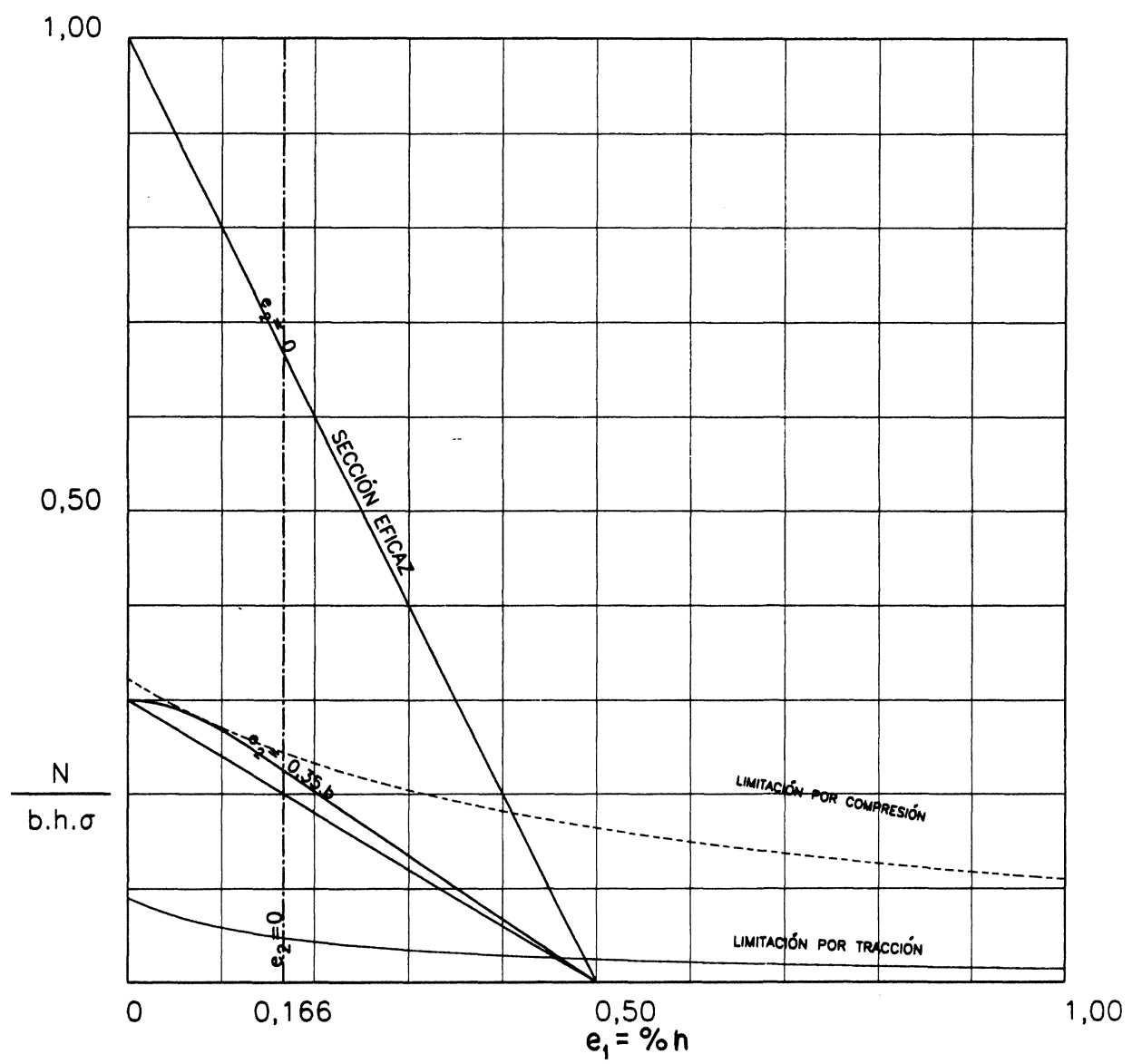

Fig. 8.- Sección rectangular sometida a compresión compuesta esviada. Valores del axil y $e_{1}$ para una serie de valores de $e_{2}$ : 
curva correspondiente al escalón de excentricidad $e_{2}$ inmediatamente superior que a la que realmente le corresponde - lo que sugiere la conveniencia de eliminar el comentariode la norma sobre el error cometido-.O, también, la escasa trascendencia que, para la resistencia de la pieza, tienen excentricidades inferiores a $0,05 \cdot b$ (Figura 9). Con las premisas anteriores se obtienen los ábacos de dimensionamiento de la pieza para las distintas excentricidades que se acompañan (Figura 10).

En la segunda colección (Figuras 11 a 13) se han obtenido los valores del axil y de la excentricidad $e_{1}$ para una excentricidad $e_{2}$ directamente relacionada con $e_{1}$ por la expresión:

$$
e_{2}=k \frac{b}{h} e_{1}
$$

calculándose los valores de axil y excentricidad para la serie de valores de $k(0 ; 0,25 ; 0,50 ; 0,75 ; 1,00)$ que se ha representado en la colección de curvas correspondiente. Cada colección incluye los valores límite obtenidos por los método de la Sección Eficaz, de la Sección Eficaz Aproximada y por los Métodos Elásticos de acuerdo con el planteamiento de FL-90 señalado anteriormente. En este conjunto de casos, las diferencias arrojadas por ambos métodos de las secciones eficaces son pequeñas.

\section{Conclusiones}

- Las fábricas son materiales fuertemente anelásticos, con una rama de resistencia a tracción muy reducida.

- Los métodos de cálculo más conservadores han excluido de los valores admisibles aquellas situaciones que implicaban la aparición de tracciones sobre la sección. FL-90, menos conservadora, llega a admitir la aparición de tensiones de tensiones de tracción de valores inferiores a la décima parte del valor de la tensión de resistencia a compresión, dentro de un comportamiento elástico de la sección.

- Las conocidas propiedades anelásticas de la fábrica han dado lugar a la aparición de distintos métodos de cálculo para este material, métodos elásticos como el método lineal, con limitación de tensiones de tracción oel método con distribución parabólica de las tensiones de compresión sobre la pieza-propuesto por D. José María Jenaro Garrido- y también métodos plásticos, como el de la Sección Eficaz. Entre ellos, la norma NBE-FL-90 recoge un método elástico y lineal con limitación de las tensiones de tracción y el método plástico de la Sección Eficaz. Este método es parcialmente recogido por el Eurocódigo 6, si bien de forma implícita.

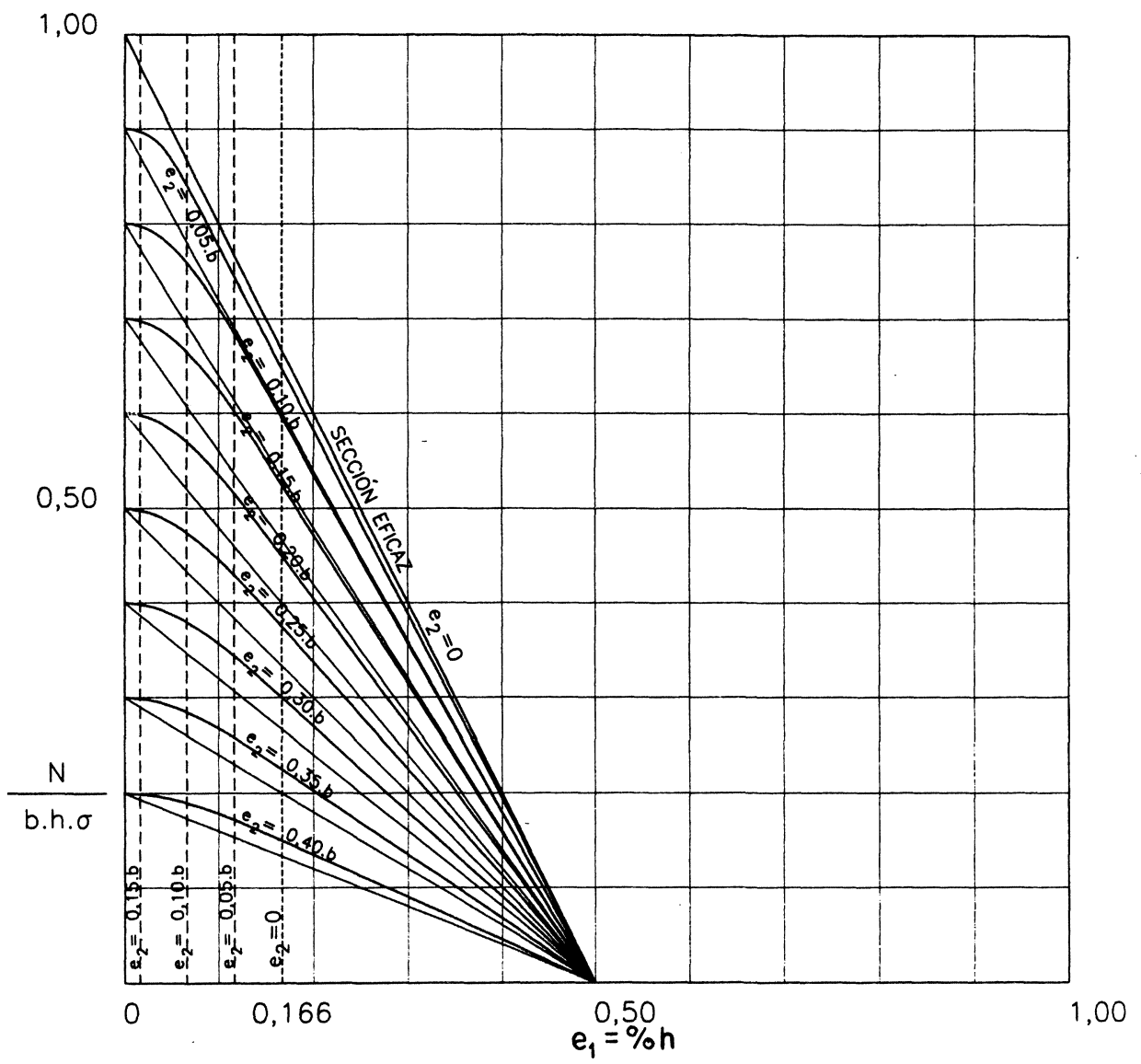

Fig. 9.- Sección rectangular sometida a compresión compuesta esviada. Valores del axil y e para una serie de valores de $e_{2}$. 


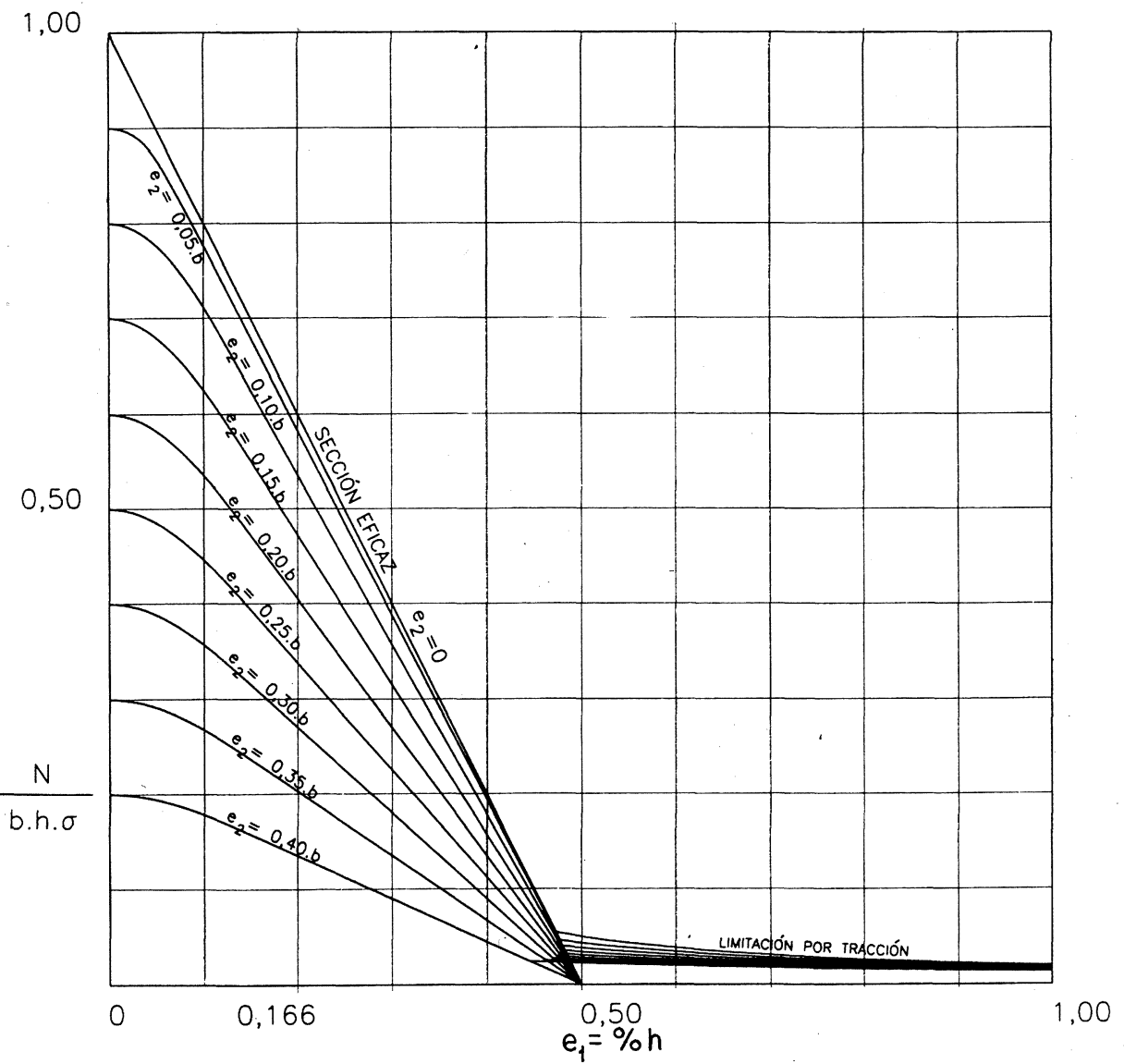

Fig. 10.- Sección rectangular sometida a compresión compuesta esviada. Valores del axil y $e_{1}$ para una serie de valores de $e_{2}$.

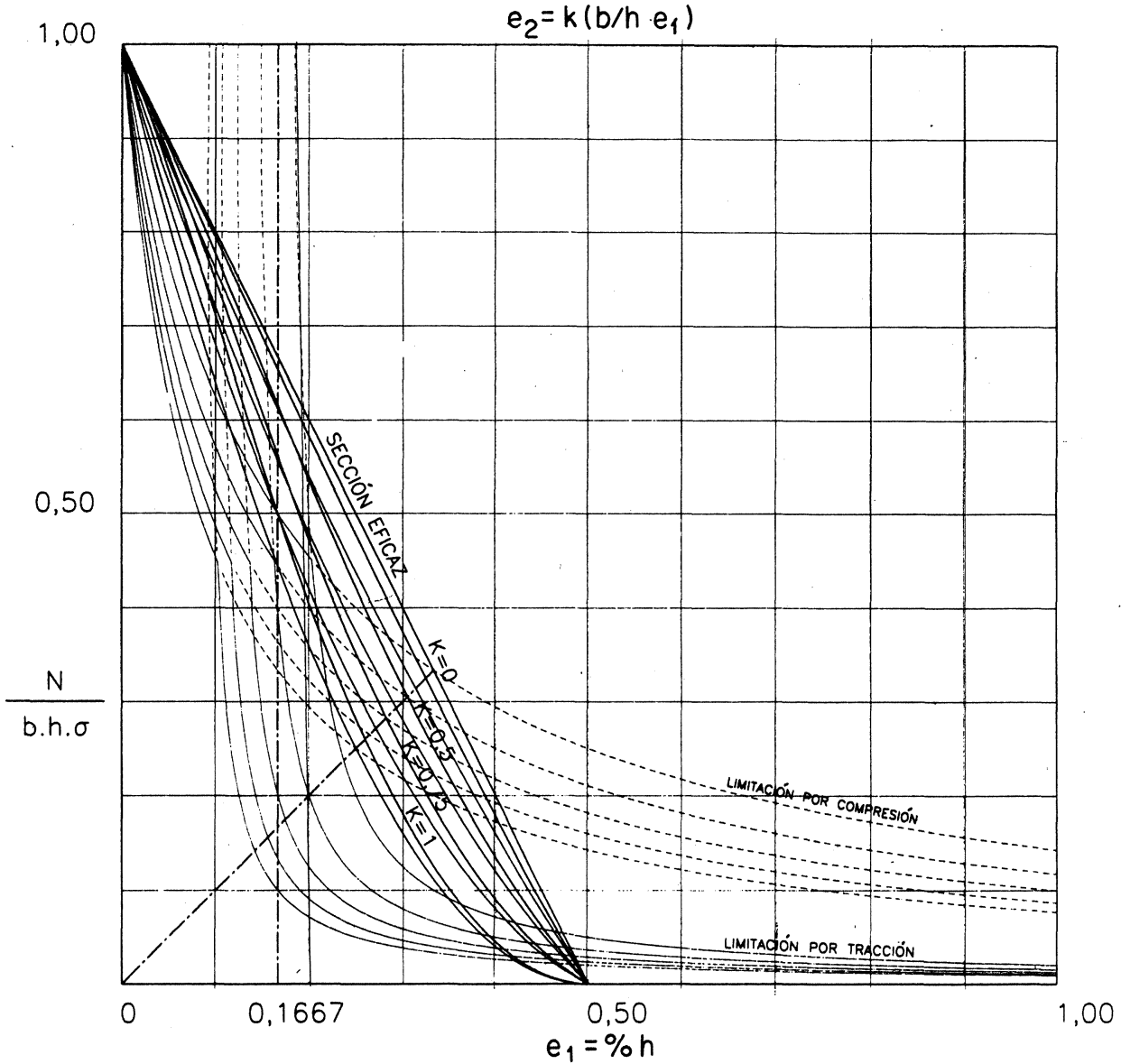

Fig. 11.- Sección rectangular sometida a compresión compuesta esviada. Valores del axil y $e_{1}$ para una excentricidad e en relación con $e_{1}$. 


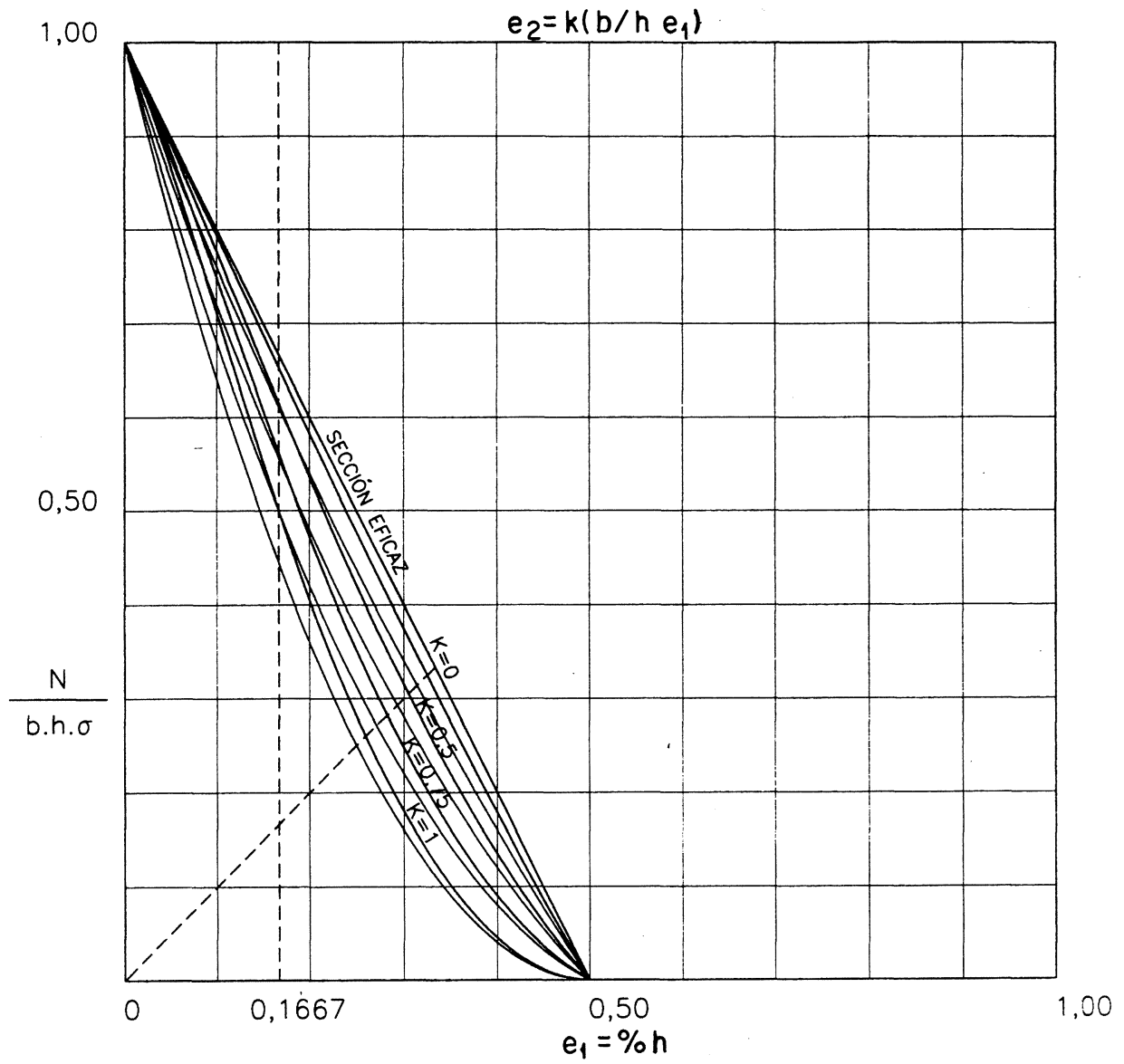

Fig. 12.- Sección rectangular sometida a compresión compuesta esviada. Valores del axil y e para una excentricidad e en relación con e . $_{2}$

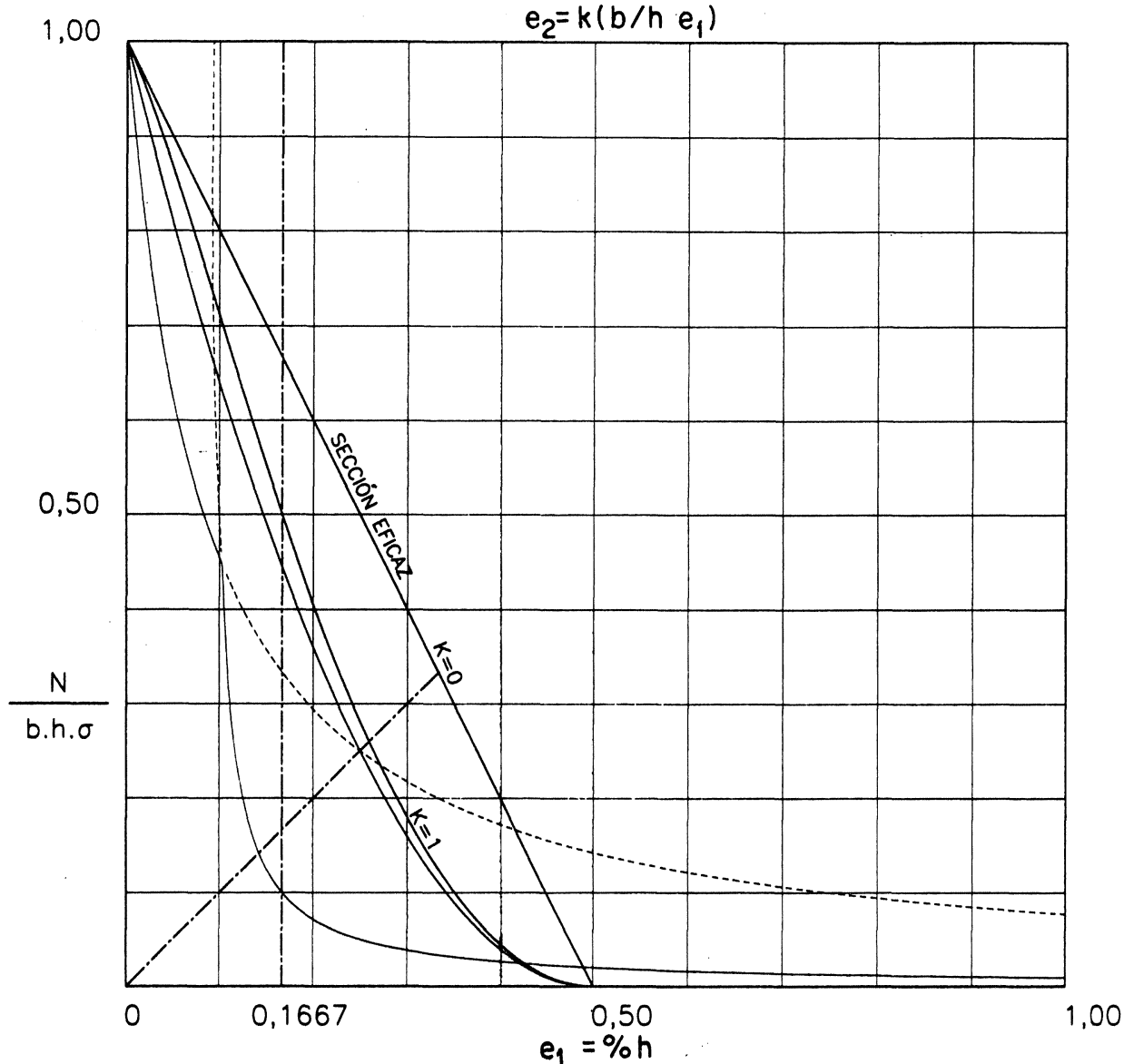

Fig. 13.- Sección rectangular sometida a compresión compuesta esviada. Valores del axil y e, para una excentricidad e, en relación con e . $_{2}$ 
- Ha parecido interesante establecer una comparación entre las situaciones de cálculo calificadas como admisibles por los distintos métodos admitidos por la normativa, para así poder elaborar unos gráficos de dimensionado de la sección que englobasen todas las situaciones que alguno de los métodos recogidos por la norma declarase como admisible.

- El método de la Sección Eficaz obliga a la determinación del tipo de sección eficaz de respuesta de la sección para cada conjunto de excentricidades $e_{1}$ y $e_{2}$. Sobre un elemento de sección rectangular se dan cinco tipos de secciones eficaces distintas: Sección Eficaz Triangular, Sección Eficaz Trapezoidal de base h, Sección Eficaz Trapezoidal de base b, Sección Eficaz Pentágono Irregular y Sección Eficaz Rectangular. Para evitar la determinación sobre qué tipo corresponde a cada situación, la norma propone el empleo de una Sección Eficaz Aproximada.

- La determinación del tipo de Sección Eficaz con la que responderá la sección para unos valores determinados de las excentricidades $e_{1}$ y $e_{2}$ en la aplicación del axil está vinculada con las coordenadas que definen el núcleo central de la pieza, salvo para el caso de la Sección Eficaz Pentágono Irregular, en la que aparecen parejas de ecuaciones de tercer grado no resolubles directamente.

- A partir de una determinada posición del axil sobre la sección es posible la determinación del tipo de Sección Eficaz resistente, de su tamañoy, por lo tanto, del valor del esfuerzo axil que agota la sección para el par de excentricidades considerado. La representación gráfica de este conjunto de valores, $e_{1}, e_{2}$ y $N_{u}$ dan lugar a las curvas de dimensionado adjuntas.

- El Método de la Sección Eficaz es mucho más permisivo que los métodos elásticos estudiados. Una excentricidad inferior a un $5 \%$ en una dirección no reduce apenas la capacidad portante de la pieza con este método. El Método de la Sección Eficaz Aproximada arroja unos valores que se aproximan a los obtenidos por el Método de la Sección Eficaz Real, con una excentricidad, en una de las direcciones, un $5 \%$ mayor.

- Los ábacos de dimensionado recogen los valores declarados como válidos por el Método de la Sección Eficaz, a los que se añade una zona de valores admisibles en función de la limitación elástica de tracción que propone la norma. Determinando el axil de la pieza y las excentricidades en su aplicación, se comprueba si el punto que representa estos valores queda en la zona admisible o no.

\section{BIBLIOGRAFÍA}

[1] "Muros Resistentes de Fábrica de Ladrillo, NBE-FL-90". Ministerio de Obras Públicas y Transportes. R.D.1723/90 de 20-12-90. B.O.E. 4-01-91

[2] "Muros Resistentes de Fábrica de Ladrillo, MV-201-72". Ministerio de la Vivienda. Madrid, 1972.

[3] Instituto Eduardo Torroja de la Construcción y el Cemento "PIET 70. Obras de Fábrica". Prescripciones del Instituto Eduardo Torroja. Madrid, 1971.

[4] "Norma UNE-ENV 1996-1-1". Norma Europea Experimental. Eurocódigo 6, Proyecto de Estructuras de Fábrica. Parte 1-1: Reglas Generales para Edificios. Reglas para Fábricay Fábrica Armada. AENOR. Madrid, 1997.

[5] JENARO GARRIDO, J.Ma : "Obras deFábrica Resistentes. Consideraciones Generales de Cálculo". Informes de la Construcción, Vol. 38, n 384 , octubre 1986.

[6] FOMBELLA GUILLÉN, RICARDO: "Estructuras de Ladrillo". UNED-Escuela de la Edificación. Madrid, 1988.

[7] HISPALYT: "El muro de ladrillo". Asociación Española deFabricantes de Ladrillo y tejas de arcilla cocida. Madrid. 1992. 